OPEN ACCESS

Edited by:

Gulisa Turashvili,

University of Toronto, Canada

Reviewed by:

Stephanie Lheureux,

Princess Margaret Hospital,

New Zealand

Mads Thomassen

Odense University Hospital, Denmark

${ }^{*}$ Correspondence:

Manuel R. Teixeira

manuel.teixeira@ipoporto.min-saude.pt

tThese authors have contributed equally to this work

Specialty section:

This article was submitted to Women's Cancer,

a section of the journal

Frontiers in Oncology

Received: 20 December 2019 Accepted: 24 June 2020 Published: 31 July 2020

Citation:

Peixoto A, Pinto P, Guerra J, Pinheiro $M$, Santos $C$, Pinto $C$, Santos R, Escudeiro C, Bartosch C, Canário R, Barbosa A, Gouveia A,

Petiz A, Abreu MH, Sousa S,

Pereira $D$, Silva $J$ and Teixeira MR (2020) Tumor Testing for Somatic and Germline BRCA1/BRCA2 Variants in

Ovarian Cancer Patients in the Context of Strong Founder Effects.

Front. Oncol. 10:1318.

doi: 10.3389/fonc.2020.01318

\section{Tumor Testing for Somatic and Germline BRCA1/BRCA2 Variants in Ovarian Cancer Patients in the Context of Strong Founder Effects}

\author{
Ana Peixoto ${ }^{1,2 \dagger}$, Pedro Pinto ${ }^{2 \dagger}$, Joana Guerra ${ }^{2}$, Manuela Pinheiro ${ }^{2}$, Catarina Santos ${ }^{1,2}$, \\ Carla Pinto ${ }^{1,2}$, Rui Santos ${ }^{2}$, Carla Escudeiro ${ }^{2}$, Carla Bartosch ${ }^{3,4}$, Rita Canário ${ }^{2,4,5,6}$, \\ Ana Barbosa ${ }^{2}$, Alfredo Gouveia ${ }^{7}$, Almerinda Petiz ${ }^{7}$, Miguel Henriques Abreu ${ }^{8}$, \\ Susana Sousa ${ }^{8}$, Deolinda Pereira ${ }^{8}$, João Silva ${ }^{1,2}$ and Manuel R. Teixeira ${ }^{1,2,9 *}$
}

${ }^{1}$ Department of Genetics, Portuguese Oncology Institute of Porto (IPO Porto), Porto, Portugal, ${ }^{2}$ Cancer Genetics Group, IPO Porto Research Center (CI-IPOP), Portuguese Oncology Institute of Porto (IPO Porto), Porto, Portugal, ${ }^{3}$ Department of Pathology, Portuguese Oncology Institute of Porto (IPO Porto), Porto, Portugal, ${ }^{4}$ Cancer Biology and Epigenetics Group, CI-IPOP, IPO Porto, Porto, Portugal, ${ }^{5}$ Epithelial Interactions in Cancer Lab, Instituto de Investigação e Inovação em Saúde (I3S)/Instituto de Patologia e Imunologia Molecular da Universidade Do Porto (IPATIMUP), University of Porto, Porto, Portugal, ${ }^{6}$ Graduate Program in Areas of Basic and Applied Biology, Instituto de Ciências Biomédicas Abel Salazar (ICBAS), University of Porto, Porto, Portugal, ${ }^{7}$ Department of Gynecology, Portuguese Oncology Institute of Porto (IPO Porto), Porto, Portugal, ${ }^{8}$ Department of Medical Oncology, Portuguese Oncology Institute of Porto (IPO Porto), Porto, Portugal, ${ }^{9}$ Institute of Biomedical Sciences Abel Salazar (ICBAS), University of Porto, Porto, Portugal

Deleterious variants in the $B R C A 1 / B R C A 2$ genes and homologous recombination deficiency (HRD) status are considered strong predictors of response to poly (ADP-ribose) polymerase (PARP) inhibitors (PARPi). The introduction of PARPi in clinical practice for the treatment of patients with advanced ovarian cancer imposed changes in the molecular diagnosis of BRCA1/BRCA2 variants. BRCA1/BRCA2 tumor testing by next-generation sequencing (NGS) can detect simultaneously both somatic and germline variants, allowing the identification of more patients with higher likelihood of benefiting from PARPi. Our main goal was to determine the frequency of somatic and germline BRCA1/BRCA2 variants in a series of non-mucinous $\mathrm{OC}$, and to define the best strategy to be implemented in a routine diagnostic setting for the screening of germline/somatic variants in these genes, including the BRCA2 c.156_157insAlu Portuguese founder variant. We observed a frequency of $19.3 \%$ of deleterious variants, 13.3\% germline, and $5.9 \%$ somatic. A higher prevalence of pathogenic variants was observed in patients diagnosed with high-grade serous ovarian cancer (23.2\%). Considering the frequencies of the c.3331_3334del and the c.2037delinsCC BRCA1 variants observed in this study $(73 \%$ of all BRCA1 pathogenic germline variants identified) and the limitations of NGS to detect the BRCA2 c.156_157insAlu variant, it might be cost-effective to test for these founder variants with a specific test prior to tumor screening of the entire coding regions of BRCA1 and BRCA2 by NGS in patients of Portuguese ancestry.

Keywords: BRCA1/BRCA2, ovarian cancer, PARPi, NGS, founder variants, tumor testing 


\section{INTRODUCTION}

Pathogenic germline variants in the breast cancer susceptibility genes $B R C A 1$ and $B R C A 2$ increase the risk for the development of ovarian cancer (OC) in carriers. The cumulative OC risk at age 80 years is 44 and $17 \%$ for $B R C A 1$ and $B R C A 2$ variant carriers, respectively (1). Women unselected for family history present germline BRCA1/BRCA2 variants in $14 \%$ of the cases when having any epithelial OC and in $\sim 17 \%$ of the cases with a high-grade serous ovarian cancer (HGSOC) diagnosis $(2,3)$. Furthermore, somatic mutations were observed in these genes in an additional $3 \%$ of HGSOC (2). In total, up to $50 \%$ of HGSOC have homologous recombination defects related with loss of function of BRCA1 or BRCA2 or other homologous recombination (HR) pathway proteins (2).

BRCA1 and BRCA2 are critical proteins in the process of $\mathrm{HR}$ repair of double-strand DNA breaks (DSBs). BRCA1/BRCA2deficient cancers are recognized as the main responders to a class of drugs known as poly (ADP-ribose) polymerase (PARP) inhibitors (PARPi) $(4,5)$. PARPi blocks the base excision repair (BER) pathway, which is involved in the repair of DNA singlestrand breaks, leading to the formation of DSBs that cannot be accurately repaired in HR-deficient cells and consequently to cell death. $(4,6)$. Deleterious variants in the BRCA1/BRCA2 genes and homologous recombination deficiency (HRD) status are strong predictors of response to PARPi (7). The PARPi olaparib (Lynparza) was the first-in-class agent to gain approval for treatment in OC by the European Medicines Agency (EMA) for use as maintenance therapy of patients with platinumsensitive relapsed, BRCA-mutated advanced epithelial ovarian, fallopian tube or primary peritoneal cancer and by the U.S. Food and Drug Administration (FDA) as monotherapy for patients with germline BRCA mutations, who have received three or more prior lines of chemotherapy (8). Consequently, it became mandatory to determine the BRCA1/BRCA2 mutational status to be able to select HGSOC patients for PARPi therapy. At that time, however, the regulatory approvals of FDA and EMA differed, as the latter also considered HGSOC patients with somatic BRCA1/BRCA2 mutations as eligible for PARPi therapy. After that, FDA and EMA approved olaparib for the maintenance treatment in the recurrent setting, regardless of BRCA status, and, more recently, in patients with newly diagnosed BRCA-mutated advanced OC. Therefore, molecular diagnosis algorithms in OC patients had to be updated, not only because of the availability of the new therapy for HGSOC, but also because molecular diagnostic labs would have to consider the detection of somatic BRCA1/BRCA2 mutations. Currently, there is no consensus regarding in which order one should undertake germline and tumor BRCA1/BRCA2 testing in HGSOC patients, but it is generally recommended to perform both $(9,10)$. Although the tumor testing strategy would need subsequent test in a blood sample of specific variants to evaluate if they are of germline or somatic origin, this would be more cost effective than performing full tumor testing after a negative full germline test to identify the rarer somatic variants. Since BRCA1/BRCA2 tumor testing can detect simultaneously both somatic and germline variants, with the exception of some variants like rearrangements, a higher number of patients who may benefit from PARPi can be identified at a faster turnaround time and at a lower cost (9).

In this study, we aimed to estimate the prevalence of germline and somatic $B R C A 1$ and $B R C A 2$ variants in a consecutive series of non-mucinous ovarian cancer patients and to evaluate the advantages and limitations of the tumor testing first strategy.

\section{MATERIALS AND METHODS}

\section{Patient Samples}

A consecutive series of patients with non-mucinous OC treated at the Portuguese Oncology Institute of Porto from January 2016 to December 2017 (135 patients), from whom formalinfixed and paraffin-embedded (FFPE) tissue and a peripheral blood sample were available, were analyzed. All patients included in the study were referred for genetic counseling and written informed consent was obtained together with collection of cancer family history and subsequent calculation of the Manchester Score, which estimates the probability of finding a germline $B R C A 1 / B R C A 2$ variant (11). Tumor samples from 10 patients with known pathogenic germline variants in BRCA1/BRCA2 were collected as validation controls. FFPE samples were obtained, with hematoxylin and eosin-stained slides carefully reviewed by an experienced pathologist in gynecological tumors, who delimited areas with $>50 \%$ tumor cells. DNA extraction was performed from tumor tissue using the cobas ${ }^{\circledR}$ DNA Sample Preparation Kit (Roche Diagnostics, Basel, Switzerland) according to the manufacturer's protocol and DNA quality was evaluated using the Qubit ${ }^{\circledR}$ 2.0 Fluorometer with the Qubit dsDNA HS Assay Kit (Thermo Fisher Scientific, Waltham, MA, USA). DNA was extracted from peripheral blood leucocytes using a standard protocol. Blood samples were used to confirm whether the variants found in the tumor samples were germline or somatic, to search for the BRCA1/BRCA2 germline founder variants and to test for large genomic rearrangements (LGRs), the latter in patients with a Manchester score equal or higher than 15.

One hundred and nine cases (80.7\%) had tumors with a pure serous histology, including 95 (70.4\%) HGSOC and 14 (10.4\%) LGSOC. Twenty-one cases (15.6\%) were of non-serous histology, including 10 (7.4\%) clear cell, nine (6.7\%) endometrioid, and two (1.5\%) mixed with clear cell, and endometrioid histology. There were also four (3\%) carcinosarcomas, and one (0.7\%) mixed carcinoma with clear cell and HGSOC components. Ninety-one FFPE samples (67.4\%) were obtained prior to treatment, 27 (20\%) post treatment with chemotherapy, and for 17 samples (12.6\%) it was not possible to obtain that information.

\section{Next-Generation Sequencing}

Next-generation sequencing (NGS) was performed in all FFPE tumor samples using the BRCA Tumor MASTR ${ }^{\mathrm{TM}}$ Plus Dx (Multiplicom, Niel, Belgium), an amplicon based NGS kit targeting the full coding sequence and adjacent intronic regions of the BRCA1/BRCA2 genes, following the manufacturer's protocol. Sequencing was carried out using a standard flow cell in the MiSeq platform (Illumina, Inc., San Diego, CA, USA) in $2 \times$ $250 \mathrm{bp}$ paired-end runs. Sequencing and bioinformatic analysis was carried out as previously described (12). All deleterious 
variants and variants of uncertain significance (VUS) identified by NGS were confirmed by Sanger sequencing following a standard protocol.

\section{Large Genomic Rearrangements and Founder Variants Screening}

The detection of BRCA1/BRCA2 LGRs and Portuguese founder variants was performed in DNA extracted from peripheral blood samples. Multiplex Ligation-dependent Probe Amplification (MLPA; MRC-Holland, Amsterdam, Netherlands) was used to detect BRCA1/BRCA2 LGRs, according to the manufacturer's instructions. Screening of the BRCA2 c.156_157insAlu variant was performed in all patients according to the protocol previously described by us (13). Screening of the BRCA1 c.2037delinsCC and c.3331_3334del variants was performed using KASPar SNP genotyping technology (LGC, Teddington, UK) on a Roche LightCycler 480 Real-Time PCR System, according to manufacturer's instructions. KASPar assay primers were designed using the Primer-BLAST design tool (14) and are available upon request. Genotyping results were analyzed using the LightCycler 480 Software 1.5.0. Positive samples were confirmed by Sanger sequencing following a standard protocol.

\section{Loss of Heterozygosity (LOH)}

VAF was used to infer biallelic inactivation by deletion of the second allele. $\mathrm{LOH}$ presence was evaluated in patients with $B R C A 1$ or BRCA2 germline pathogenic variants and VUS that were called in a heterozygous state in the tumor samples. $\mathrm{LOH}$ was considered present when the germline BRCA1/BRCA2 VAF was $>60 \%$, and/or at least two informative (heterozygous) single nucleotide variants (SNVs) showed a VAF $<0.4$ or $>0.6$ (15).

\section{RESULTS}

\section{Variant Detection}

A total of 10 FFPE tumor DNA samples from OC patients with known pathogenic germline variants were used to validate the NGS assay, including the bioinformatic analysis. This sample set included deletions, duplications, point mutations, and the BRCA2 c.156_157insAlu Portuguese founder variant (Table 1), which is not detectable using standard sequencing methodologies in FFPE samples (12). Regarding the known germline point mutations, the concordance between Sanger sequencing in peripheral blood samples and the NGS-based tumor test on FFPE samples was $100 \%$ (8/8). As expected, the germline BRCA2 c.156_157insAlu variant was not called by the NGS tumor assay pipeline described above.

The NGS-based tumor test was performed in 136 ovarian tumor samples derived from 135 patients. We detected 27 pathogenic variants in 26 patients (19.3\%; Table 2): 16 patients with a deleterious $B R C A 1$ variant (61.5\%) and 10 patients with a deleterious BRCA2 variant (38.5\%). A total of 18 (13.3\%) patients had germline variants (11 in the BRCA1 gene and seven in the BRCA2 gene) and eight (5.9\%), including one patient with two pathogenic variants in the BRCA1 gene, presented mutations that were found to be somatic (five in BRCA1 and three in $B R C A 2)$. The most frequent deleterious variant was the BRCA1 c.3331_3334del, detected in $4.4 \%(6 / 135)$ of the tumors and representing $22.2 \%$ of the pathogenic variants found in this series. This variant together with c.2037delinsCC represents $73 \%(8 / 11)$ of all the $B R C A 1$ pathogenic germline variants identified.

We also detected 12 VUS in 11 patients (8.1\%). Within this group, eight (5.9\%) patients had a germline VUS and four (3\%) patients had a somatic VUS (Table 3). One patient had one VUS in each of the genes, one somatic BRCA1 VUS and one germline BRCA2 VUS.

In one of the samples, no deleterious variant was identified using the variant filters previously described. However, when reviewing the data, a pathogenic variant (BRCA1 c.1459_1463delinsTAT) with a $4 \%$ VAF was identified that had been discarded by the software due to low VAF $(<5 \%)$. This tumor sample was obtained post neoadjuvant chemotherapy (paclitaxel and carboplatin) and another available sample, prior to treatment, was subsequently analyzed. The same BRCA1 pathogenic mutation was detected in the second analysis, but now with a $19 \%$ VAF.

\section{LOH}

$\mathrm{LOH}$ was evaluated in patients with $B R C A 1$ or $B R C A 2$ germline pathogenic variants ( $n=11$ for $B R C A 1 ; n=6$ for $B R C A 2$ ) and VUS ( $n=1$ for BRCA $1 ; n=7$ for BRCA2) in the tumor samples. In the sample with BRCA2 c.156_157insAlu, LOH was not possible to evaluate since this variant was not called by the software and there were no informative SNVs. The subset of germline pathogenic variants had a mean VAF of $80 \%$ and $69 \%$ for BRCA1 and BRCA2 genes, respectively. The subset of germline VUS had a mean VAF of $53 \%$. We considered that LOH occurred in 10 out of 11 patients (91\%) and in 4 out of 6 patients (67\%) with a BRCA1 and BRCA2 germline pathogenic variant, respectively. Loss of the wild type allele was not observed in the tumor from the patient with a germline $B R C A 1$ VUS. In patients with germline BRCA2 VUS, loss of the wild type allele was seen in $29 \%(2 / 7)$ of the tumor samples.

\section{Manchester Score}

The Manchester score was calculated for 133 patients (three patients belonged to the same family) and a median score of 15 was obtained. The median score was 14 for patients where no germline pathogenic variants or VUS were identified $(n=107)$, 15 for patients with a germline VUS ( $n=8$, Table 3 ), and 21 for patients with a germline pathogenic variant $(n=18$, Table 2$)$.

\section{Frequency of Mutations by Histology}

A higher prevalence of pathogenic variants was observed in patients diagnosed with HGSOC, namely 17 of 95 (17.9\%) patients with germline variants and 22 of 95 (23.2\%) patients with germline/somatic variants. Four additional tumors, out of the 40 with other histologies (10\%), had a deleterious germline or somatic BRCA mutation, namely 2 of 9 (22.2\%) endometrioid carcinomas, both of which were high grade, and 2 of $4(50 \%)$ carcinosarcomas. 
TABLE 1 | Known pathogenic germline variants used to validate the NGS assay.

\begin{tabular}{|c|c|c|c|c|c|c|}
\hline Gene & HGVS coding & HGVS protein & Tumor & Blood & RD tumor & VAF tumor $\%$ \\
\hline BRCA1 & c.3331_3334del & p.(Gln1111AsnfsTer5) & Positive & Positive & 2,812 & 62 \\
\hline BRCA1 & c.2490_2497dup & p.(Leu833CysfsTer16) & Positive & Positive & 457 & 86 \\
\hline BRCA1 & c.2086dup & p.(Thr696AsnfsTer16) & Positive & Positive & 1,247 & 70 \\
\hline BRCA1 & c. $5278-1 \mathrm{G}>\mathrm{T}$ & & Positive & Positive & 840 & 71 \\
\hline BRCA1 & c.3331_3334del & p.(Gln1111AsnfsTer5) & Positive & Positive & 534 & 80 \\
\hline BRCA1 & c.2906del & p.(Asn969llefsTer31) & Positive & Positive & 5,917 & 85 \\
\hline BRCA2 & c.156_157insAlu & & Negative & Positive & & \\
\hline BRCA2 & c.156_157insAlu & & Negative & Positive & & \\
\hline
\end{tabular}

$R D$, read depth; $V A F$, variant allele frequency.

\section{DISCUSSION}

The National Authority of Medicines and Health Products (Infarmed) approved olaparib in Portugal as maintenance therapy only in HGSOC patients with a germline or somatic BRCA mutation. Therefore, it became important to evaluate whether a tumor-testing-first strategy would be the most costeffective option, allowing for the simultaneous detection of both germline and somatic BRCA1/BRCA2 variants. However, the detection of somatic mutations depends on DNA extraction from FFPE tumor material, which is usually of poor quality and highly fragmented. Additionally, tumor samples are very heterogeneous and contamination with DNA from normal tissue is often an issue. In order to detect somatic mutations in addition to the germline variants, it is necessary to use a methodology with high sensitivity and specificity, such as the use of NGS after tumor macrodissection of the tumor areas marked by a pathologist. However, accurate detection of LGRs in tumor samples with NGS is not straightforward. Moreover, the specific variant c.156_157insAlu represents about $50 \%$ of the $B R C A 2$ pathogenic variants identified in the Portuguese population, but it is not detected by standard sequencing technologies neither by common bioinformatic approaches using NGS data (12). In this study, we used Multiplicom BRCA MASTR Dx assay for the detection of $B R C A 1 / B R C A 2$ variants using DNA extracted from FFPE tumor samples, for which it has CE-IVD marking. Furthermore, our bioinformatic analysis used Sophia Genetics software which also obtained CE-IVD marking. Our main goal was to determine the frequency of somatic and germline $B R C A 1 / B R C A 2$ variants in a series of non-mucinous OC, and to define the best strategy to be implemented in a routine diagnostic setting for screening of germline/somatic variants in these genes, including the BRCA2 c.156_157insAlu founder variant.

The first task of this work consisted in the analysis of FFPE tumor DNA samples from OC patients with known pathogenic germline variants to validate the NGS assay. All germline point mutations were detected by the NGS-based tumor test, allowing us to implement this technique in a routine diagnostic setting.
However, as expected, the germline BRCA2 c.156_157insAlu variant was not called by the NGS tumor assay pipeline, using the software Sophia $\mathrm{DDM}^{\circledR}$, in two tumor samples from the validation series. Taking this into account, blood samples from the 135 patients were analyzed to search for the BRCA2 c.156_157insAlu germline variant. In this study, we detected the presence of germline pathogenic variants in $13.3 \%$ of the 135 patients studied, which is comparable to previous studies. The frequency of $B R C A 1$ and $B R C A 2$ germline variants in women with ovarian cancer varies in the literature (6-41\%), with the lowest prevalence observed in unselected series of patients with OC (16-18). A higher prevalence of $B R C A 1 / B R C A 2$ variants $(>15 \%)$ has been consistently described in patients with $\operatorname{HGSOC}(16,19)$. Although we observed a predominance $(23.2 \%)$ of BRCA1/BRCA2 variants in patients with HGSOC, these alterations were not exclusively associated with this group, as they were also frequently found in carcinomas with other histologies $(10 \%)$. These findings corroborate those obtained by Pennington et al. (20), which found HR gene variants (germline and somatic) to be also common in carcinomas with non-HGSOC histologies. In this work, we identified a $B R C A 1 / B R C A 2$ deleterious variant frequency of $50 \%(2 / 4)$ in ovarian carcinosarcomas. Although this frequency can be overestimated due to the limitation of a small sample size, the association of ovarian carcinosarcomas with BRCA1/BRCA2 pathogenic variants has already been described in the literature (20-22), including two (17\%) out of 12 ovarian carcinosarcomas, one with a germline and the other with a somatic mutation. Indeed, there are a few studies indicating that ovarian carcinosarcomas and HGSOC may arise from the same precursor lesion in the Fallopian tube (serous intraepithelial carcinoma) (23).

The identification of specific and recurrent/founder variants in any given population allows a more efficient and costsaving mutational screening approach. In our previous work, we demonstrated that two variants in BRCA1 (c.2037delinsCC and c.3331_3334del) and one in BRCA2 (c.156_157insAlu) together represent about $50 \%$ of all deleterious variants found in Portuguese hereditary breast and ovarian cancer families mostly originated from northern Portugal (13). These data allowed us to define our current strategy of starting the 
TABLE 2 | Pathogenic variants identified.

\begin{tabular}{|c|c|c|c|c|c|c|c|c|c|}
\hline Patient & Histological type & Gene & HGVS coding & HGVS protein & Tumor & Blood & $\begin{array}{l}\text { RD } \\
\text { tumor }\end{array}$ & $\begin{array}{c}\text { VAF tumor } \\
\%\end{array}$ & MS \\
\hline 1 & HGSOC & $B R C A 1$ & c.1192_1193del & p.(Ser398ThrfsTer2) & Positive & Negative & 5,885 & 52 & 15 \\
\hline 3 & HGSOC & $B R C A 1$ & c. $1058 \mathrm{G}>\mathrm{A}$ & p.(Trp353Ter) & Positive & Positive & 5,170 & 55 & 15 \\
\hline 4 & HGSOC & $B R C A 1$ & c. 2037 delinsCC & p.(Lys679AsnfsTer4) & Positive & Positive & 12,302 & 63 & 28 \\
\hline 7 & HGSOC & $B R C A 1$ & c.3331_3334del & p.(Gln1111AsnfsTer5) & Positive & Positive & 6,015 & 92 & 18 \\
\hline 8 & HGSOC & $B R C A 1$ & c.3331_3334del & p.(Gln1111AsnfsTer5) & Positive & Positive & 6,118 & 86 & 28 \\
\hline 9 & HGSOC & $B R C A 1$ & c.3331_3334del & p.(Gln1111AsnfsTer5) & Positive & Positive & 7,995 & 87 & 17 \\
\hline 10 & HGSOC & $B R C A 1$ & c.3331_3334del & p.(Gln1111AsnfsTer5) & Positive & Positive & 3,544 & 84 & 31 \\
\hline 11 & HGSOC & $B R C A 1$ & c.3331_3334del & p.(Gln1111AsnfsTer5) & Positive & Positive & 4,700 & 71 & 19 \\
\hline 15 & HGSOC & $B R C A 1$ & c. $4485-2 A>C$ & & Positive & Negative & 3,610 & 6 & \\
\hline 25 & HGSOC & $B R C A 1$ & c. $116 \mathrm{G}>\mathrm{T}$ & p.(Cys39Phe) & Positive & Negative & 30,150 & 24 & 18 \\
\hline 16 & HGSOC & BRCA2 & c. $8488-1 G>A$ & & Positive & Positive & 2,838 & 49 & 23 \\
\hline 17 & HGSOC & $B R C A 2$ & c.5073dup & p.(Trp1692MetfsTer3) & Positive & Positive & 6,900 & 78 & 23 \\
\hline 18 & HGSOC & BRCA2 & c.4964dup & p.(Tyr1655Ter) & Positive & Positive & 3,592 & 80 & 12 \\
\hline 19 & HGSOC & $B R C A 2$ & c.5073dup & p.(Trp1692MetfsTer3) & Positive & Positive & 9,183 & 78 & 19 \\
\hline 20 & HGSOC & $B R C A 2$ & c.4964dup & p.(Tyr1655Ter) & Positive & Positive & 2,708 & 70 & 20 \\
\hline 21 & Endometrioid & $B R C A 2$ & c.5436del & p.(Glu1812AspfsTer3) & Positive & Negative & 5,638 & 69 & 19 \\
\hline 22 & HGSOC & $B R C A 2$ & c.5950_5961delinsTGCT & p.(Lys1984CysfsTer16) & Positive & Negative & 21,046 & 65 & 15 \\
\hline 23 & HGSOC & $B R C A 2$ & c.9379_9400del & p.(Trp3127AlafsTer29) & Positive & Negative & 1,357 & 6 & 18 \\
\hline
\end{tabular}

HGSOC, high-grade serous ovarian cancer; RD, read depth; VAF, variant allele frequency; MS, Manchester score.

genetic study of all families by testing these variants before the screening of the entire coding regions of the BRCA1 and $B R C A 2$ genes. In this study, we identified six patients with the BRCA1 c.3331_3334del (4.4\%), two with the BRCA1 c.2037delinsC variant (1.5\%), and one patient with the BRCA2 c.156_157insAlu variant (0.7\%). Together, these three variants represent 50\% (9/18) of all germline deleterious variants found in this series, indicating that it might be cost-effective to test for these founder variants with a specific test prior to tumor screening of the entire coding regions of $B R C A 1$ and $B R C A 2$ by NGS in patients of Portuguese ancestry (Figure 1). Furthermore, since the detection of LGRs by NGS in FFPE samples, including the BRCA2 c.156_157insAlu variant, is not yet optimized due to the low quality of FFPE samples and the possibility of somatic chromosomal deletions and gains that might shield germline LGRs, blood samples from these patients must be collected to test for this specific variant and for germline LGRs [which are relatively rare in our population (13)] at least in patients with a Manchester score higher than 14. Nevertheless, any strategy for the detection of BRCA1 and BRCA2 variants must be adapted to specific populations, considering the presence and nature of recurrent and/or founder variants and the ability of current methodologies to detect them in FFPE tissue. In the future, it might be time-saving to optimize the bioinformatics pipeline to detect all variant types in FFPE tissue, making the blood sample only necessary to determine the eventual germline origin of a variant identified in the initial tumor testing by NGS (eventually preceded by founder variant testing in the tumor if considered cost-effective).

In this study, Manchester score was determined for 133 patients. The median of this score was higher for patients with pathogenic germline variants in comparison to patients where no germline variants or a VUS was identified, reflecting that a family history of breast and/or ovarian cancer increases significantly the chance of identifying women with a germline $B R C A 1 / B R C A 2$ variant. In general, a $10 \%$ estimated probability of finding a germline $B R C A 1 / B R C A 2$ variant is considered to be cost-effective for DNA testing (24). Although a strong family history increases the chance of identifying these variants, it has been reported that family history may be absent in a significant percentage of germline BRCA1/BRCA2 variant carriers (25). Recently, an overall probability of a germline BRCA1/BRCA2 variant above $10 \%$ was described for all women with epithelial OC (26). Therefore, it is recommended to refer 
TABLE 3 | Variants of unknown significance identified.

\begin{tabular}{|c|c|c|c|c|c|c|c|c|}
\hline Patient & Histological type & Gene & HGVS coding & HGVS protein & Tumor & Blood & VAF tumor $\%$ & MS \\
\hline 26 & HGSOC & BRCA1 & c. $898 \mathrm{G}>\mathrm{A}$ & p.(Glu300Lys) & Positive & Negative & 19 & 12 \\
\hline 27 & HGSOC & BRCA1 & c. $994 \mathrm{C}>\mathrm{T}$ & p.(Arg332Trp) & Positive & Positive & 19 & 15 \\
\hline 29 & HGSOC & BRCA1 & c.5420T>G & p.(lle1807Ser) & Positive & Negative & 38 & 13 \\
\hline 30 & Clear cell & BRCA2 & c. $19 \mathrm{G}>\mathrm{C}$ & p.(Glu7Gln) & Positive & Negative & 15 & 10 \\
\hline 31 & HGSOC & BRCA2 & c. $1343 G>A$ & p.(Arg448His) & Positive & Positive & 49 & 21 \\
\hline 23 & HGSOC & BRCA2 & c.6351_6377del & p.(Val2118_Cys2126del) & Positive & Negative & 69 & \\
\hline 26 & HGSOC & BRCA2 & c. $7435+6 \mathrm{G}>\mathrm{A}$ & & Positive & Positive & 56 & \\
\hline 35 & HGSOC & BRCA2 & c. $8036 \mathrm{~A}>\mathrm{G}$ & p.(Asp2679Gly) & Positive & Positive & 50 & 13 \\
\hline 36 & LGSOC & BRCA2 & c. $8902 A>G$ & p.(Thr2968Ala) & Positive & Positive & 50 & 14 \\
\hline 37 & HGSOC & BRCA2 & c.9364G>C & p.(Ala3122Pro) & Positive & Positive & 85 & 27 \\
\hline
\end{tabular}

HGSOC, high-grade serous ovarian cancer; LGSOC, low grade serous ovarian cancer; VAF, variant allele frequency; MS, Manchester score.

all women with these tumors for genetic risk evaluation and DNA analysis. In the present series, however, only 1 out of $18(6 \%)$ patients with a BRCA1/BRCA2 pathogenic germline variant had a prior probability lower than $10 \%$. Although BRCA variant testing for ovarian cancer patients must be done in the context of targeted therapy to estimate the potential clinical benefit, in our population the majority of patients with a germline BRCA variant would have been identified based on personal and family history of breast and/or ovarian cancer, revealing that the current model for genetic testing based in risk assessment using familial risk models is still an accurate tool to select patients for germline genetic testing in our population.

It is still not entirely clear if the magnitude of benefit from PARPi for a patient with OC harboring BRCA1/BRCA2 somatic mutations is the same as for those with a germline variant (27). Phase 3 trials that included germline and somatic BRCA mutated patients revealed similar outcomes between these two groups $(28,29)$. Somatic mutations were ascertained in several studies, with report rates varying from 4 to $7 \%(20,30)$. Our study revealed the presence of somatic $B R C A 1 / B R C A 2$ pathogenic mutations in $5.9 \%$ of the 135 patients studied and in $30.8 \%$ of all the patients with pathogenic variants, which is comparable to previous studies. Testing both tumor and blood samples increased the proportion of pathogenic variants identified in OC patients from 13.3 to $19.3 \%$ ( 17.9 to $23.2 \%$ in patients with HGSOC), allowing the identification of more patients with higher likelihood of benefiting from PARPi.

Several factors can influence the number of variants detected in a tumor. A factor that must be taken into account is the quality of FFPE samples for DNA analysis (9). For instance, the age of the FFPE block has a significant impact on the quality and the number of variants detected (31). Despite the limited number of studies evaluating the mutation profile in pre- and post-chemotherapy OC specimens, tumor mutational shifts have been described after chemotherapy (32). This phenomenon may be due to pre-existing intra-tumoral heterogeneity and sampling bias, cytotoxic therapy applying selective pressure, or direct druginduced genetic aberrations (32). One patient from our series was tested in two different samples, one obtained prior to and the other after treatment with chemotherapy. No mutations were detected in the post-treatment sample using the cutoff of a minimum 5\% VAF, although a BRCA1 pathogenic mutation was present with a $4 \%$ VAF. In the sample that was obtained prior to treatment, the same $B R C A 1$ pathogenic variant was present with a VAF of $19 \%$. This finding highlights the importance of selecting the most suitable sample for BRCA1/BRCA2 tumor testing in OC patients. Although the analysis of metastatic tissue at the time of progression may provide a more accurate indication of tumors likely to respond to PARPi treatment, the information available from clinical trials relates to the analysis of primary ovarian tumors (9). While there are no recommendations about the timing of BRCA1/BRCA2 mutation analysis concerning preand post-therapeutic tumor samples, an adequate collection of tumor samples with a high tumor content prior to surgery is advised (9).

It is accepted that tumors with $B R C A 1 / B R C A 2$ germline pathogenic variants usually exhibit $\mathrm{LOH}$, resulting from deletion of the wild type allele, which can be inferred from the high VAF of the mutant allele. BRCA locus-specific $\mathrm{LOH}$ in germline $B R C A 1 / B R C A 2$ carriers has been associated with sensitivity to DNA damaging agents. In a recent work, absence of $\mathrm{LOH}$ was observed in $7 \%$ of BRCA1 and $16 \%$ of BRCA2 ovarian tumors and was correlated with decreased overall survival in ovarian cancers treated with platinum chemotherapy (33). In this study, LOH was observed in 91 and $67 \%$ of ovarian tumors with a $B R C A 1$ and $B R C A 2$ germline pathogenic mutation, respectively, which is in accordance with previous reports. Given that most ovarian tumors with germline BRCA deleterious variants show $\mathrm{LOH}$ and loss of the wildtype allele in tumor tissue provides strong evidence for a deleterious germline mutation, we can use $\mathrm{LOH}$ status to provide some evidence about the clinical significance of VUS in ovarian cancer tumors. Whereas, $\mathrm{LOH}$ was observed in more than $80 \%$ of the patients with BRCA1/BRCA2 germline pathogenic variants, loss of the 


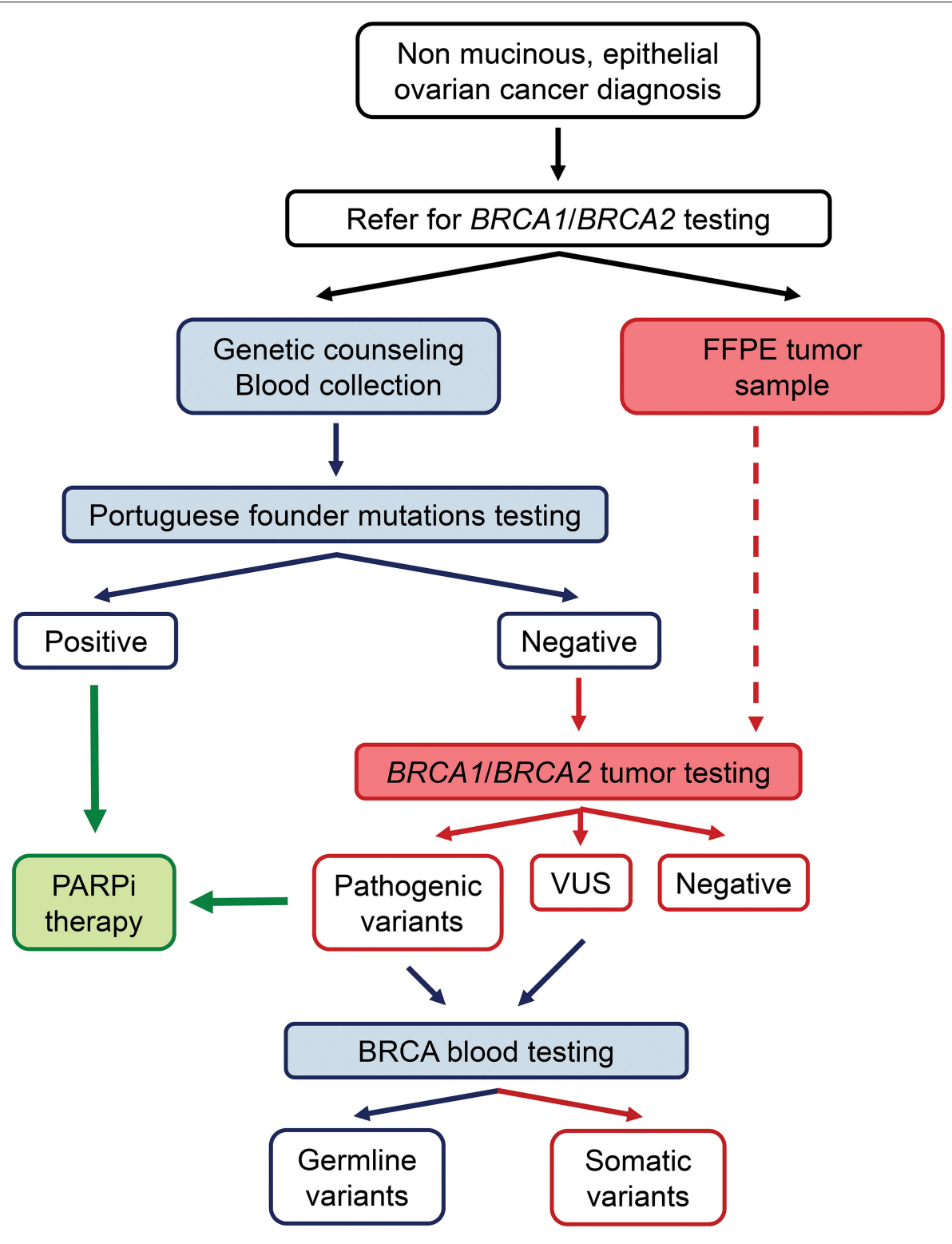

FIGURE 1 | Strategy for detection of germline and somatic BRCA1/BRCA2 mutations in ovarian cancer patients: A specific blood test for the detection of the three most common mutations in our population is performed before tumor screening of the entire coding regions of $B R C A 1$ and $B R C A 2$ by NGS. Blood samples from these patients are used to confirm whether the variants found in the tumor samples are germline or somatic. Patients with pathogenic variants in $B R C A 1 / B R C A 2$ genes are eligible for PARPi therapy.

wild type allele was observed in about $25 \%$ of the patients with BRCA1/BRCA2 VUS. These results suggest that most of the germline VUS identified are probably not pathogenic. One of these variants is the c.994C $>\mathrm{T}$ in the BRCA1 gene, which is described in ClinVar (ID 55775) as a VUS and was detected in the tumor sample with a VAF of 19\%, which is relatively low for a germline variant. On the other hand, the BRCA2 c. $9364 \mathrm{G}>\mathrm{C}$ variant was identified with a VAF of $85 \%$, which is suggestive of $\mathrm{LOH}$ and pathogenicity. Nevertheless, $\mathrm{LOH}$ might be the result of genomic instability, therefore, additional studies will be required to further characterize these variants.

In conclusion, we have characterized the mutation spectrum of $B R C A 1 / B R C A 2$ in a consecutive series of ovarian carcinomas, observing a frequency of $19.3 \%$ of deleterious variants, $13.3 \%$ germline, and $5.9 \%$ somatic. Considering the frequencies of the variants observed in our study and the limitations of NGS, we recommend performing a specific blood test for the detection of the three most common variants in our population prior to tumor 
screening of the entire coding regions of BRCA1 and BRCA2 by NGS. Any deleterious variant identified in the tumor testing, which by itself is predictive of better response to PARPi, should subsequently be evaluated for its germline or somatic origin.

\section{DATA AVAILABILITY STATEMENT}

The original contributions presented in the study are publicly available. This data can be found here: European Nucleotide Archive (ENA) with accession number PRJEB38270 (https:// www.ebi.ac.uk/ena/data/view/PRJEB38270).

\section{ETHICS STATEMENT}

Ethical review and approval was not required for the study on human participants in accordance with the local legislation and institutional requirements. The patients/participants provided their written informed consent to participate in this study.

\section{REFERENCES}

1. Kuchenbaecker KB, Hopper JL, Barnes DR, Phillips KA, Mooij TM, Roos-Blom MJ, et al. Risks of breast, ovarian, and contralateral breast cancer for BRCA1 and BRCA2 mutation carriers. JAMA. (2017) 317:240216. doi: 10.1001/jama.2017.7112

2. Cancer Genome Atlas Research N. Integrated genomic analyses of ovarian carcinoma. Nature. (2011) 474:609-15. doi: 10.1038/nature10166

3. Norquist BM, Brady MF, Harrell MI, Walsh T, Lee MK, Gulsuner $\mathrm{S}$, et al. Mutations in homologous recombination genes and outcomes in ovarian carcinoma patients in GOG 218: an NRG oncology/gynecologic oncology group study. Clin Cancer Res. (2018) 24:777-83. doi: 10.1158/1078-0432.CCR-17-1327

4. Bryant HE, Schultz N, Thomas HD, Parker KM, Flower D, Lopez E, et al. Specific killing of BRCA2-deficient tumours with inhibitors of poly(ADPribose) polymerase. Nature. (2005) 434:913-7. doi: 10.1038/nature03443

5. Farmer H, McCabe N, Lord CJ, Tutt AN, Johnson DA, Richardson TB, et al. Targeting the DNA repair defect in BRCA mutant cells as a therapeutic strategy. Nature. (2005) 434:917-21. doi: 10.1038/nature03445

6. Ashworth A. A synthetic lethal therapeutic approach: poly(ADP) ribose polymerase inhibitors for the treatment of cancers deficient in DNA double-strand break repair. J Clin Oncol. (2008) 26:3785-90. doi: 10.1200/JCO.2008.16.0812

7. Ray-Coquard I, Pautier P, Pignata S, Perol D, Gonzalez-Martin A, Berger R, et al. Olaparib plus bevacizumab as first-line maintenance in ovarian cancer. N Engl J Med. (2019) 381:2416-28. doi: 10.1056/NEJMoa1911361

8. Ledermann J, Harter P, Gourley C, Friedlander M, Vergote I, Rustin G, et al. Olaparib maintenance therapy in patients with platinum-sensitive relapsed serous ovarian cancer: a preplanned retrospective analysis of outcomes by BRCA status in a randomised phase 2 trial. Lancet Oncol. (2014) 15:85261. doi: 10.1016/S1470-2045(14)70228-1

9. Capoluongo E, Ellison G, Lopez-Guerrero JA, Penault-Llorca F, Ligtenberg MJL, Banerjee S, et al. Guidance statement on BRCA1/2 tumor testing in ovarian cancer patients. Semin Oncol. (2017) 44:187-97. doi: 10.1053/j.seminoncol.2017.08.004

10. Konstantinopoulos PA, Norquist B, Lacchetti C, Armstrong D, Grisham RN, Goodfellow PJ, et al. Germline and somatic tumor testing in epithelial ovarian cancer: ASCO guideline. J Clin Oncol. (2020) 38:122245. doi: 10.1200/JCO.19.02960

\section{AUTHOR CONTRIBUTIONS}

APei, PP, and JG performed experiments. APei, PP, JG, MP, CS, CP, RS, and CE analyzed data. CB, RC, AB, AG, APet, MA, SS, DP, and JS provided samples and data. APei, PP, and MT wrote the manuscript. MT designed and supervised the study. All authors critically revised and approved the manuscript.

\section{FUNDING}

PP, JG, MP, and RC were research fellows of the Fundação para a Ciência e a Tecnologia (FCT; UID/DTP/0076/POCI01-0145-FEDER-006868, SFRH/BD/138670/2018, SFRH/BPD/ $113014 / 2015$, and $\mathrm{PB} / \mathrm{BD} / 128001 / 2016$, respectively). $\mathrm{AB}$ was a research fellow supported by the project NORTE01-0145-FEDER-000027, supported by Norte Portugal Regional Operational Programme (NORTE 2020), under the PORTUGAL 2020 Partnership Agreement, through the European Regional Development Fund (ERDF). CE and RS were research fellows of the Núcleo Regional do Norte da Liga Portuguesa Contra o Cancro.

11. Evans DG, Harkness EF, Plaskocinska I, Wallace AJ, Clancy T, Woodward ER, et al. Pathology update to the manchester scoring system based on testing in over 4000 families. J Med Genet. (2017) 54:674-81. doi: 10.1136/jmedgenet-2017-104584

12. Pinto P, Peixoto A, Santos C, Rocha P, Pinto C, Pinheiro M, et al. Analysis of founder mutations in rare tumors associated with hereditary breast/ovarian cancer reveals a novel association of BRCA2 mutations with ampulla of vater carcinomas. PLoS ONE. (2016) 11:e0161438. doi: 10.1371/journal.pone.0161438

13. Peixoto A, Santos C, Pinto P, Pinheiro M, Rocha P, Pinto C, et al. The role of targeted BRCA1/BRCA2 mutation analysis in hereditary breast/ovarian cancer families of Portuguese ancestry. Clin Genet. (2015) 88:41-8. doi: $10.1111 /$ cge.12441

14. Ye J, Coulouris G, Zaretskaya I, Cutcutache I, Rozen S, Madden TL. PrimerBLAST: a tool to design target-specific primers for polymerase chain reaction. BMC Bioinformatics. (2012) 13:134. doi: 10.1186/1471-2105-13-134

15. de Jonge MM, Ruano D, van Eijk R, van der Stoep N, Nielsen $\mathrm{M}$, Wijnen JT, et al. Validation and implementation of BRCA1/2 variant screening in ovarian tumor tissue. J Mol Diagn. (2018) 20:600-11. doi: 10.1016/j.jmoldx.2018.05.005

16. Alsop K, Fereday S, Meldrum C, deFazio A, Emmanuel C, George J, et al. BRCA mutation frequency and patterns of treatment response in BRCA mutation-positive women with ovarian cancer: a report from the Australian ovarian cancer study group. J Clin Oncol. (2012) 30:265463. doi: 10.1200/JCO.2011.39.8545

17. Moslehi R, Chu W, Karlan B, Fishman D, Risch H, Fields A, et al. BRCA1 and BRCA2 mutation analysis of 208 ashkenazi jewish women with ovarian cancer. Am J Hum Genet. (2000) 66:1259-72. doi: 10.1086/302853

18. Soegaard M, Kjaer SK, Cox M, Wozniak E, Hogdall E, Hogdall C, et al. BRCA1 and BRCA2 mutation prevalence and clinical characteristics of a populationbased series of ovarian cancer cases from Denmark. Clin Cancer Res. (2008) 14:3761-7. doi: 10.1158/1078-0432.CCR-07-4806

19. Schrader KA, Hurlburt J, Kalloger SE, Hansford S, Young S, Huntsman DG, et al. Germline BRCA1 and BRCA2 mutations in ovarian cancer: utility of a histology-based referral strategy. Obstet Gynecol. (2012) 120(2 Pt. 1):23540. doi: 10.1097/AOG.0b013e31825f3576

20. Pennington KP, Walsh T, Harrell MI, Lee MK, Pennil CC, Rendi $\mathrm{MH}$, et al. Germline and somatic mutations in homologous recombination genes predict platinum response and survival in ovarian, 
fallopian tube, and peritoneal carcinomas. Clin Cancer Res. (2014) 20:764-75. doi: 10.1158/1078-0432.CCR-13-2287

21. Carnevali IW, Cimetti L, Sahnane N, Libera L, Cavallero A, Formenti G, et al. Two cases of carcinosarcomas of the ovary involved in hereditary cancer syndromes. Int J Gynecol Pathol. (2017) 36:64-70. doi: 10.1097/PGP.0000000000000290

22. Sonoda Y, Saigo PE, Federici MG, Boyd J. Carcinosarcoma of the ovary in a patient with a germline BRCA2 mutation: evidence for monoclonal origin. Gynecol Oncol. (2000) 76:226-9. doi: 10.1006/gyno.1999.5681

23. Ardighieri L, Mori L, Conzadori S, Bugatti M, Falchetti M, Donzelli CM, et al. Identical TP53 mutations in pelvic carcinosarcomas and associated serous tubal intraepithelial carcinomas provide evidence of their clonal relationship. Virchows Arch. (2016) 469:61-9. doi: 10.1007/s00428-016-1933-x

24. NICE Guidelines [CG164]. Familial Breast Cancer: Classification, Care and Managing Breast Cancer and Related Risks in People With a Family History of Breast Cancer (2017). Available online at: https://www.nice.org.uk/guidance/ cg164.

25. Eccles DM, Balmana J, Clune J, Ehlken B, Gohlke A, Hirst C, et al. Selecting patients with ovarian cancer for germline BRCA mutation testing: findings from guidelines and a systematic literature review. Adv Ther. (2016) 33:12950. doi: 10.1007/s12325-016-0281-1

26. Arts-de Jong M, de Bock GH, van Asperen CJ, Mourits MJ, de Hullu JA, Kets CM. Germline BRCA1/2 mutation testing is indicated in every patient with epithelial ovarian cancer: a systematic review. Eur J Cancer. (2016) 61:137-45. doi: 10.1016/j.ejca.2016.03.009

27. George A, Kaye S, Banerjee S. Delivering widespread BRCA testing and PARP inhibition to patients with ovarian cancer. Nat Rev Clin Oncol. (2017) 14:284-96. doi: 10.1038/nrclinonc.2016.191

28. Coleman RL, Oza AM, Lorusso D, Aghajanian C, Oaknin A, Dean $A$, et al. Rucaparib maintenance treatment for recurrent ovarian carcinoma after response to platinum therapy (ARIEL3): a randomised, double-blind, placebo-controlled, phase 3 trial. Lancet. (2017) 390:1949-61. doi: 10.1016/S0140-6736(17)32440-6
29. Mirza MR, Monk BJ, Herrstedt J, Oza AM, Mahner S, Redondo A, et al. Niraparib maintenance therapy in platinum-sensitive, recurrent ovarian cancer. N Engl J Med. (2016) 375:2154-64. doi: 10.1056/NEJMoa16 11310

30. Cunningham JM, Cicek MS, Larson NB, Davila J, Wang C, Larson MC, et al. Clinical characteristics of ovarian cancer classified by BRCA1, BRCA2, and RAD51C status. Sci Rep. (2014) 4:4026. doi: 10.1038/srep04026

31. Van Heetvelde M, Van Bockstal M, Poppe B, Lambein K, Rosseel T, Atanesyan $\mathrm{L}$, et al. Accurate detection and quantification of epigenetic and genetic second hits in BRCA1 and BRCA2-associated hereditary breast and ovarian cancer reveals multiple co-acting second hits. Cancer Lett. (2018) 425:12533. doi: 10.1016/j.canlet.2018.03.026

32. Tan SH, Sapari NS, Miao H, Hartman M, Loh M, Chng WJ, et al High-throughput mutation profiling changes before and 3 weeks after chemotherapy in newly diagnosed breast cancer patients. PLoS ONE. (2015) 10:e0142466. doi: 10.1371/journal.pone.0142466

33. Maxwell KN, Wubbenhorst B, Wenz BM, De Sloover D, Pluta J, Emery $\mathrm{L}$, et al. BRCA locus-specific loss of heterozygosity in germline BRCA1 and BRCA2 carriers. Nat Commun. (2017) 8:319. doi: 10.1038/s41467-01700388-9

Conflict of Interest: The authors declare that the research was conducted in the absence of any commercial or financial relationships that could be construed as a potential conflict of interest.

Copyright (c) 2020 Peixoto, Pinto, Guerra, Pinheiro, Santos, Pinto, Santos, Escudeiro, Bartosch, Canário, Barbosa, Gouveia, Petiz, Abreu, Sousa, Pereira, Silva and Teixeira. This is an open-access article distributed under the terms of the Creative Commons Attribution License (CC BY). The use, distribution or reproduction in other forums is permitted, provided the original author(s) and the copyright owner(s) are credited and that the original publication in this journal is cited, in accordance with accepted academic practice. No use, distribution or reproduction is permitted which does not comply with these terms. 